\title{
Hybrid Surgical Hot Clinic (HSHC): Evaluation of Surgical Hot Clinic Services during COVID-19 Lockdown
}

\author{
Adeel Abbas Dhahri ${ }^{1}$ (D) Raheel Ahmad ${ }^{2} \cdot$ Bilal Fazal Shaikh $^{3} \cdot$ Olubunmi Sajinyan $^{4} \cdot$ Ibrahim Warrag $^{1}$. \\ Maitreyi Patel $^{1} \cdot$ Bogdan Ivanov $^{1}$
}

Accepted: 24 January 2021 / Published online: 7 February 2021

(C) Société Internationale de Chirurgie 2021

\begin{abstract}
Background Surgical Hot Clinic (SHC) is an acute, ambulatory service for management provided on an outpatient basis. Following the start of global Novel Coronavirus (COVID-19) pandemic and as per the statement released by the Association of Surgeons of Great Britain and Ireland (ASGBI), we also modified our services to hybrid SHC (HSHC) by mainly providing telephonic follow-up with an occasional face-to-face (F2F) service. We conducted a service evaluation to assess the effectiveness and serviceability of HSHC during a pandemic.

Methods This service evaluation was conducted from 30th March till 26th May 2020. The pathway was developed to mostly telephonic consultation with selective face-to-face consultation at a designated area in the medical ambulatory unit. The analysis then performed using SPSS version 21.

Results As the overall attendance fell in hospital, 149 patients, including 54(36.2\%) male, and 95(63.8\%) females, attended SHC during COVID-19 lockdown. Out of these 149, 87(58.3\%) were referred from Accident \& Emergency (A\&E), 2(1.3\%) from GP, 9(6.04\%) after scan through radiology department, while 51(34.2\%) after discharge from hospital. Out of those who have telephonic consultation $(n=98), 12$ patients were called in for review with either blood tests or further clinical examination. In total, only 10 out of 149 patients required admission to the hospital, for either intervention or symptomatic treatment.

Conclusion Hybrid Surgical Hot Clinic (HSHC) with both telephonic \& face-to-face consultation, as per requirement, is flexible, effective and safe patient-focused acute surgical service during COVID-19 like crisis.
\end{abstract}

\section{Introduction}

The concept of ambulatory care is very wide in medical field. The Royal College of Emergency Medicine has defined ambulatory care as 'acute clinical care which includes investigation, treatment and rehabilitation for

Adeel Abbas Dhahri

adeelabbas.dhahri@nhs.net

1 The Princess Alexandra Hospital NHS Trust, Harlow, UK

2 John Radcliffe Hospital, Oxford, UK

3 Manchester Royal Infirmary, Oxford street, Manchester, UK

4 Essex Partnership University Trust, Harlow, UK whom admission to hospital would have been the default option'. Surgical Hot Clinic (SHC) is a patient-focused surgical ambulatory care, within hospital emergency service, for consultation and management provided on an outpatient basis [1-3]. This service has been successfully running in National Health Services (NHS) hospitals and here at the Princess Alexandra Hospital NHS Trust UK, a district hospital at the outskirts of London, for quite sometimes now. Here at our hospital, it is mainly senior registrar-based service with secondary consultant-based care [4].

It has already been proved in the commissioning guide, released by the Royal College of Surgeons of England, and other researches that SHC can effectively reduce 
unnecessary admissions as well as a financial burden on the hospital while providing safe care after senior review. Such care can be directed to the management of abdominal pain, biliary colic, cholecystitis, and uncomplicated diverticulitis following vigilant patient selection [1, 5-9].

Since the World Health Organisation (WHO) declared Novel Coronavirus (COVID-19) as a Public Health Emergency of International Concern (PHEIC), many changes adopted around the world including changes to work pattern and use of telemedicine in acute care setting $[10,11]$. As per the statement released by the Association of Surgeons of Great Britain and Ireland (ASGBI) in March 2020, we also introduced changes within our emergency services to continue to provide safe and non-stop acute patient care [12]. We modified our SHC services by developing a local pathway to mainly provide telephonic follow-up while using Medical Ambulatory unit as additional SHC service for face-to-face (F2F) attendance.

We hypothesize that use of telephonic service in SHC is safer and as efficient as face-to-face consultation. To confirm this, we aim to assess patient care service provision during a crisis like COVID-19 pandemic at the Princess Alexandra Hospital NHS Trust, to identify feasibility and safety.

\section{Material and methods}

The Surgical Hot Clinic (SHC) service at the Princess Alexandra Hospital NHS Trust is based in the surgical assessment unit (SAU) as an identified quality improvement project, since 2017 with an average 250 attendance per month in addition to on-call surgical referrals. A Senior Registrar leads this service as per rota, supported by the oncall consultant. Sources of patient referrals include Accident \& Emergency (A\&E), GP referrals, Radiology department and Post-discharge early review. It runs from 0800 till 1700, seven days a week.

\section{Intervention}

A service evaluation on Surgical Hot Clinic services was carried out at the Princess Alexandra Hospital NHS Trust UK, from $30^{\text {th }}$ March till $26^{\text {th }}$ May 2020, during a period of lockdown in the United Kingdom [13]. Approval was taken from local hospital Information Governance (IG) \& Audit Departments with registration number 134 . To protect patients and healthcare staff members from contacting COVID-19, a local pathway for the patients suitable for SHC created and most of the services changed to telephonic consultation with selective F2F service. A designated area of Medical Ambulatory unit was used for F2F consultation, where availability of Personal Protective
Equipment (PPE) and social distancing were made possible. This service evaluation examined the provision of SHC after incorporating telephonic consultation, during times of COVID-19 crisis, according to local and national guidelines.

\section{Modified local hybrid SHC pathway (Fig. 1)}

Usual activity of SHC was completely suspended, to avoid an unnecessary patient visit to the hospital and waiting time. Further, the following measures were made. By default, all patients were offered a telephonic consultation. Telephonic consultation covered all the essential questions required for usual consultation. If requiring only blood tests, patients were diverted to the main A\&E area. This group of patients were advised to go back home and wait for surgical registrar telephonic consultation with results. Only selected patients for F2F review were called in Medical Ambulatory unit for a consultation;

1 Referrals from GP \& A\&E

All the GP's referrals were assessed on telephonic discussions with GP and diverted to A\&E only for further assessment. All A\&E referrals received and reviewed by Surgical On-Call team in A\&E. After Review, the patient could go straight to telephonic consultation via radiology.

2 Radiology Follow-up

All the patients after the scan, except those indicated by the radiologist, were requested to go back to home to avoid long exposure in the hospital while waiting for the report. They were advised to wait for senior surgical registrar telephonic call and radiology department then to update the SHC registrar to chase the report of the investigation. During the consultation, patients were inquired about the symptoms and informed about the scan report. Depending upon the telephonic consultation outcome, patients were advised for further telephonic or F2F consultation or return to the hospital for further management. All patients were also offered telephonic follow-up consultation in $24 \mathrm{~h}$.

3 Ward discharges Follow-up

Patients in this group were either simple ward discharges requiring early review or repeat the blood tests or postoperative patients. Unless necessary, all patients were advised not to return to the hospital unless asked to do so for the F2F consultation.

\section{Measures}

Inclusion criteria for telephonic consultation were same as pre-lockdown period. However, inclusion criteria for F2F consultation included patients requiring clinical 


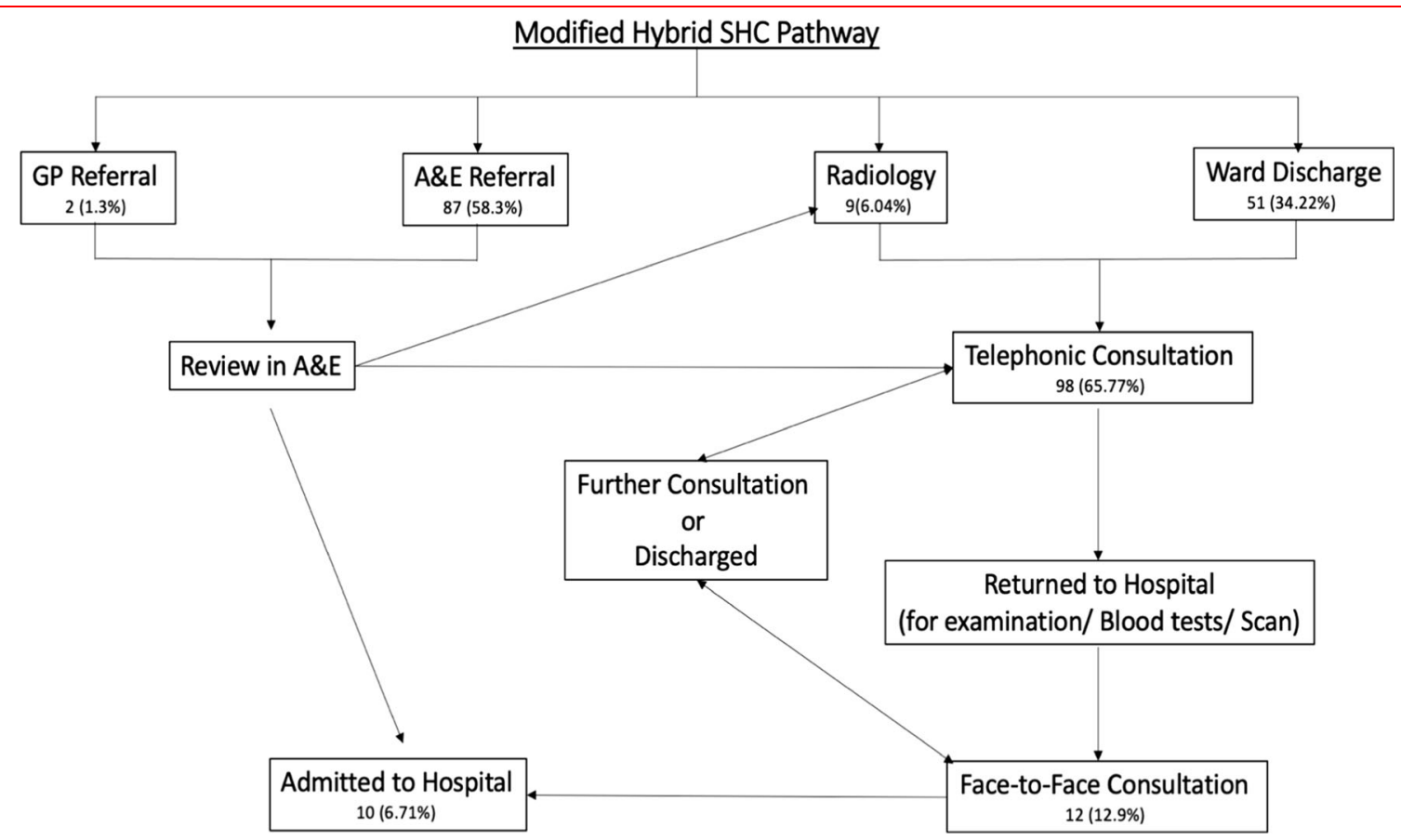

Fig. 1 Hybrid SHC Pathway

examination, repeat blood tests, positive findings on the investigations requiring admission or further assessment. Exclusion criteria for included paediatric patients of age less than 18 and negative investigation results.

\section{Analysis}

During data collection, demographic information was collected. Other variables included a source of referral to SHC, a reason for referral, type of service offered, diagnosis made, whether admitted or not, type of investigation offered, an incidence of re-admission and whether patient referred to another department or not. All the data were collected on collated excel data sheet explicitly designed for this service evaluation. Data analysis was performed using SPSS version 20.

As this study was retrospective assessment of clinical services through hospital audit department (Registration \# 134), formal ethical approval was not required.

SQUIRE 2.0 reporting guideline was followed to report this service evaluation [14].

\section{Results}

\section{Pre-COVID lockdown 'SHC'}

During pre-COVID lockdown period (from 1st January till 15th March 2020) 477 patient including 239 (50.1\%) were male, while 238 (49.9\%) were female. All of these patients had face-to-face appointments/ consultations as default. $81 / 477$ (16.98\%) were admitted mostly for conservative management (59/81) for acute illnesses like diverticulitis, diabetic foot with peripheral vascular disease (PVD), biliary pathology including acute cholecystitis, biliary colic, choledocholithiasis and acute pancreatitis of any severity. 22 out of those 81 admitted patients required interventions like incision $\&$ drainage of abscesses (9/22), appendicectomies $(11 / 22)$, and umbilical or inguinal hernia repair (1 each).

As lockdown started, overall A\&E attendance at our hospital fell from 9500 per month on average to 4484 during the lockdown. The overall number of A\&E referrals to surgical On-Call team also dropped from 400 on average to 194 .

\section{During COVID lockdown 'HSHC'}

\section{Sources of referrals}

From 30th March till 26th May 2020, all the one hundred and forty-nine patients deemed suitable for SHC were included in this service evaluation. Out of them, 54 (36.2\%) were males, while $95(63.8 \%)$ females. There was no missing data found.

Total 87 (58.3\%) referred from A\&E, 2 (1.3\%) from GP, $9(6.04 \%)$ after radiological follow-ups and $51(34.22 \%)$ after discharge from hospital. Out of these discharge 
follow-ups, 46 were discharged from the ward, one sent home through Patient at Home $(\mathrm{P} @ \mathrm{H})$ service from the ward, and 4 were postoperative follow-ups (Fig. 1).

\section{Consultation type}

A total $98(65.77 \%)$ had telephonic consultation, while 51 $(34.23 \%)$ were called in for F2F consultation. Out of those $98(65.77 \%)$ who had telephonic consultation, only 12 $(12.9 \%)$ were called in for review (Fig. 1). Out of these, two patients came for the blood test, and ten patients were called for further clinical or wound examination. From telephonic consultations, one patient had six telephonic follow-ups and was found to be the only maximum number of follow-up in the study. The reason for maximum followup was found to be a requirement of serial investigations through a multi-disciplinary approach. A maximum number of follow-ups during F2F consultation was 3, noted in 4 patients. Two patients did not respond to the telephonic consultation and were considered as failed appointments, and their GPs were updated for safeguarding. When compared, telephonic with F2F consultation, the earlier group had no significant difference in terms of early identification of complications or complaints. Out of 98 patients of telephonic consultation group, only 12 required hospital visits. Out of these 12 patients, 9 needed clinical examination assessment where telephonic consultation was not deemed safe, 1 required wound review, while 2 needed repeat blood tests. Only 2 of these 12 patients ended up being admitted for either incision and drainage of perianal abscess or conservative management of acute cholecystitis. Such results clearly show that telephonic consultation is very much safely doable.

\section{Presenting complaint and diagnosis}

Most presenting complaints dealt with were abdominal pain $(116,77.85 \%)$, painful lump in natal cleft $(4,2.7 \%)$, bleeding PR $(4,2.7 \%)$, and wound discharge $(4,2.7 \%)$ (Table 1).

Most common diagnosis was biliary tree pathology 34 $(22.81 \%)$, acute appendicitis $27(18.1 \%)$ and non-specific abdominal pain $26(17.4 \%)$. Other common diagnosis encountered were acute diverticulitis in $8(5.4 \%)$ patients, and $7(4.69 \%$ ) had abscesses (like perianal or pilonidal abscesses).

\section{Management plans}

Most patients were treated as outpatient with regular telephonic follow-ups in the acute setting for patient safety. Only 12(8.05\%) out of 149 required admissions. However, two refused for admission because of ongoing COVID-19
Table 1 Complaints

\begin{tabular}{ll}
\hline Complaints & $n, \%$ \\
\hline Abdominal Pain & $114,76.5$ \\
Painful Lump at Natal Cleft & $4,2.7$ \\
Peri-anal pain & $3,2.0$ \\
Post-op Review & $3,2.0$ \\
PR Bleed & $4,2.7$ \\
Reduced Stoma Output & $2,1.3$ \\
Wound Discharge & $4,2.7$ \\
Breast Pain \& Lump & $3,2.0$ \\
Abdominal Pain \& PR Bleed & $1,0.7$ \\
Abdominal Pain \& PV Bleed & $1,0.7$ \\
Abdominal Lump & $1,0.7$ \\
Back pain & $1,0.7$ \\
Diarrhoea & $1,0.7$ \\
Discoloration of left foot & $1,0.7$ \\
GORD & $1,0.7$ \\
Left Leg pain & $1,0.7$ \\
Leg cellulitis & $1,0.7$ \\
Orbital Haematoma & $1,0.7$ \\
Pain in Groin & $1,0.7$ \\
Pain in Toes & $1,0.7$ \\
\hline &
\end{tabular}

PR $=$ Per Rectum, GORD = Gastro-Oesophageal Reflux Disorder $\mathrm{SHC}=$ Surgical Hot Clinic, $\mathrm{GP}=$ General Practitioner, $\mathrm{A} \& \mathrm{E}=\mathrm{Ac}-$ cident and Emergency

fear and were regularly followed-up for safety-netting (Fig. 1). Out of those $10(6.71 \%)$ who were successfully admitted, 5 required surgical interventions like appendicectomies, incision \& drainage of abscesses and femoral hernia repair. One patient required Interventional Radiology (IR) drainage of the sub-hepatic abscess, one had Endoscopic Retrograde Cholangiopancreatography (ERCP) for choledocholithiasis, while the remaining 3 were admitted only for symptomatic treatment (Table 2).

It was also noticed that 25 out of 27 diagnosed patients of appendicitis were successfully treated conservatively using hybrid SHC services. Out of 34 patients of biliary pathology, only two required admission. In this group of biliary pathology, 23 were managed for acute cholecystitis, 5 with biliary colic, 5 with choledocholithiasis and one with mild pancreatitis. Through this hybrid SHC, we successfully managed acute diverticulitis in all 8 patients, 2 with haemorrhoids, 2 with epiploic appendagitis, 5 with abscesses or cellulitis, and 3 patients of PVD.

A total of $29(19.46 \%)$ patients were referred to further specialities, after review and diagnosis, like Gastroenterology department in 11 patients, 5 patients to Gynaecology department, 2 patients to the Breast clinic, and 3 were referred to Medicine. 
Table 2 Clinical Outcomes

\begin{tabular}{ll}
\hline Outcomes & $n, \%$ \\
\hline Appendicectomy & $2,1.3$ \\
ERCP & $1,0.7$ \\
Hernia Repair & $1,0.7$ \\
I\&D & $2,1.3$ \\
IR Drainage & $1,0.7$ \\
Conservative Tx or Discharges & $141,94.6$ \\
Referred to Tertiary Centre & $1,0.7$
\end{tabular}

ERCP = Endoscopic Retrograde Cholangiopancreatography, $\mathrm{I} \& \mathrm{D}=$ Incision and Drainage, IR = Interventional Radiology, $\mathrm{Tx}=$ Treatment

\section{Unintended consequences}

None of the 149 patients required re-admission, nor reported to $\mathrm{A} \& \mathrm{E}$ or Resus room during their management through hybrid SHC. No mortality was observed.

\section{Discussion}

Concept of ambulatory care is not new in medicine and surgery, and the guidelines are available from the Royal Colleges of both Emergency Medicine and Surgery United Kingdom (UK) [1-3]. With recent coronavirus COVID-19 pandemic, the Association of Surgeons of Great Britain and Ireland (ASGBI) released guidelines on the delivery of emergency general surgery service in the UK to accommodate safe care for the acutely ill patients [11, 12]. We also followed the guidelines and drafted a bespoke local pathway for SHC to make it work as a hybrid service for acutely ill ambulatory surgical patients (Fig. 1). The pathway helped to understand the process of care of the acute surgical patient during the current crisis with a potential to avoid an un-necessary waiting time for the patient till the scan is reported [15]. This, in turn, reduces the risk of infection transmission among patient and healthcare workers. As the world's response to COVID-19 pandemic evolves, such a strategy can be effectively utilised in future with safe and better patient outcome.

The important concept of telemedicine of avoiding chances of virus transmission between patient and surgeon to improve safe patient practice has been widely accepted at the Princess Alexandra Hospital NHS Trust at a larger level [16-18]. During evaluation, we also found that SHC patients also benefited with distant consultation using a telephonic form of telemedicine with only 12 patients requiring admissions to hospital which is comparable to our admission rate before COVID-19 lockdown period.
Like in our study, A\&E is the main reason for attendance in SHC, required to reduce the waiting time in the A\&E, averting pressure, and improve services for better patient experience as well as satisfaction [5, 19]. If pathway accurately followed, GP and patient experience can also be improved by diverting referrals to SHC. In addition, any postoperative patient, as ward follow-up, who need urgent review when clinical services are overbooked, can benefit from such service, especially during times of crisis [9].

Most common complaint encountered during SHC assessment remains abdominal pain secondary to nonspecific abdominal pain, biliary problems and mild diverticulitis. Other common presentations for SHC attendance include abscesses [1, 7]. For this reason, different surgical sub-specialities can utilise the effectiveness of SHC services. Although an effective route to avoid unnecessary admissions to the hospital, as evident in literature, SHC attending patients may require admission or re-admission to the hospital depending upon their clinical condition or results of the investigation [5]. As mentioned earlier, no readmissions, presentations in $\mathrm{A} \& \mathrm{E}$ or Resus room nor mortality observed. Furthermore, none of the patients made any complaint or expressed frustration against HSHC service provision. This further confirms the safer practice of SHC during this hybrid approach.

In a safer and designated environment of ambulatory medical unit, clinical examination, blood investigations or acute management can be carried out whereas SHC keeps pace with limited F2F activity during a crisis like current COVID-19. NHS England has recently published clinical guidelines for the selective use of F2F appointment whenever deemed to do so [20]. This can bring a variability in an acute care setting by first F2F followed by telephonic followups rather than $\mathrm{F} 2 \mathrm{~F}$ unless required, a different approach to a standard A\&E practice. In our study, a selective F2F consultation was chosen as an alternative route, for safer consultation where telephonic consultation was not deemed to provide appropriate care. As an outline, our study proved that the telephonic consultation had no significant difference in terms of early identification of complications or complaints when compared with a F2F consultation.

It is proven in the literature that being cost-effective telemedicine can efficiently reduce health-care cost significantly [17]. Financial benefits of our model are difficult for us to quantify during current pandemic, especially regarding the cost of PPE per patients, when the government provided all the supplies. However, it is established that cost of PPE increased to up to sixfold during the peak of COVID-19 crisis [21]. Patients were trying to avoid coming to hospital during that time of lockdown; at the same time, we were avoiding admissions. All these patients who had telephonic consultation could have cost us at least 
one day of admission, thus, a financial and health-care burden.

To the best of our knowledge, no study to date has utilised additional provision of telemedicine in SHC. SHC proved to be a flexible acute surgical service, when telemedicine can be incorporated. This model could continue after the pandemic, avoiding crowding in A\&E and parking fees for the patients, for example. Our study data also proved that it did work well for the patients by different departments engagements. Our study also highlighted advantages like applicability, safer patient management and staff wellbeing. If appropriately driven, it provides services to the patient with better, safer and meaningful outcomes.

\section{Limitation}

This was an initial pilot study with smaller sample size. There is a possibility of variation between doctors' approach to managing patients, reflected by the number of times consultation provided, further highlighting limitations. We plan to perform a furthermore significant study with more numbers and changes, as Hybrid Surgical Hot Clinic has the potential to work even after COVID-19 pandemic.

\section{Conclusion}

This service evaluation has shown that Surgical Hot Clinic (SHC) service can be effectively and safely modified to a hybrid service of a telephonic and F2F consultation, during a major crisis like COVID-19, without comprising patient safety and staff well-being. We do also suggest further efforts to endure and continue this model of HSHC after pandemic. A further large-scale study is required to understand long-term practical implementations, its limitations and further use in clinical care.

\section{Compliance with ethical standards}

Conflict of interest The authors declare that they have no conflict of interest or funding.

Ethical approval As this study was retrospective assessment of clinical services through hospital audit department (Registration \# 134), formal ethical approval was not required.

\section{References}

1. The Royal College of Surgeons of England, Emergency General Surgery-Commissioning Guide, The Royal College of Surgeon of England, 2014. https://www.rcseng.ac.uk/library-and-publica tions/rcs-publications/docs/emergency-general-guide/
2. The Royal College of Emergency, Medicine Ambulatory Emergency Care Toolkit 2019. https://www.rcem.ac.uk/docs/RCEM\% 20Guidance/RCEM_Ambulatory_Emergency_Care_Toolkit_ (Feb_2019).pdf

3. Torcivia A, Vons C, Barrat C et al (2011) Influence of mesh type on the quality of early outcomes after inguinal hernia repair in ambulatory setting controlled study: Glucamesh ${ }^{\circledR}$ vs Polypropylene ${ }^{\circledR}$. Langenbeck's Arch Surg 396:173-178

4. Hubbard T, Thomas R (2014) Improving the surgical hot clinic. BMJ Qual Improv Rep 3(u206573):w202639

5. Ryan M, Kelliher G, Mealy K et al (2017) Characteristics of patients who are acutely admitted to hospital under surgical care and do not have a surgical procedure - Is there an alternative to admission? Surgeon 15:259-266

6. Th'ng F, Skouras C, Paterson-Brown A et al (2017) Emergency general surgery "Hot Clinics" reduce admission rates and duration of inpatient stay. Frontline Gastroenterol 8:53-61

7. Anderson I. The Future of Emergency General Surgery: a joint document, 2015. https://www.acpgbi.org.uk/content/uploads/ 2016/07/Future-of-EGSjoint-document_Iain-Anderson_140915. pdf

8. Patel N, Ravindran R, Paterson-Brown S (2020) Emergency general surgery 'Hot Clinic': Efficiency, prevention of hospital admissions and factors influencing patient experience. Surgeon 18:e39-e46

9. Imtiaz M, Sreelekha A, Shatkar V (2016) Surgical Hot ClinicAn Effective Pathway of Reducing Emergency Admissions and the Associated Costs. Int J Healthcare Sci 4:198-205

10. World Health Organisation, Coronavirus Disease (COVID-19)events as they happen, 2020. https://www.who.int/emergencies/ diseases/novelcoronavirus-2019/events-as-they-happen

11. Guerci C, Maffioli A, Bondurri AA et al (2020) COVID-19: How can a department of general surgery survive in a pandemic? Surgery 167:909-911

12. Association of Surgeons of Great Britain \& Ireland. Delivering the EmergencyGeneral Surgery service in the UK during the coronavirus COVID-19 pandemic, 2020. https://www.asgbi.org. $\mathrm{uk} /$ news/2020/mar/20/delivering-the-emergency-general-surgeryservice-in-the-uk-during

13. When did the UK lockdown start and how long will it last?, Heart, 2020. https://www.heart.co.uk/news/how-long-uk-lock down-last/

14. Ogrinc G, Davies L, Goodman D et al (2016) SQUIRE 2.0 $(<\mathrm{em}>$ Standards for QUality Improvement Reporting Excellence) $</$ em $>$ : revised publication guidelines from a detailed consensus process. BMJ Qual Safety 25:986-992

15. Lai W, Gutteridge C, Regan A et al (2017) The Value of a Surgical Assessment Unit Ultrasound Facility. BMJ Qual Improv Rep 6:u209155.w3729. https://doi.org/10.1136/bmjquality. u209155.w3729

16. Wherton J, Shaw S, Papoutsi C et al (2020) Guidance on the introduction and use of video consultations during COVID-19: important lessons from qualitative research. BMJ Leader 4:120-123

17. Chou E, Hsieh YL, Wolfshohl J et al (2020) Onsite telemedicine strategy for coronavirus (COVID-19) screening to limit exposure in ED. Emerg Med J 37(6):335-337

18. Dhahri AA, Iqbal MR, Pardoe H (2020) Agile Application of Video Telemedicine During the COVID-19 Pandemic. Cureus 12:e11320

19. Mohamed MS, Mufti GR (2005) The surgical assessment uniteffective strategy for improvement of the emergency surgical pathway? J R Soc Med 98:14-17

20. Getting It Right First Time, NHS Improvement Clinical guide for the management of remote consultations and remote working in secondary care during the coronavirus pandemic, NHS England 
and NHS Improvement, 2020. https://www.england.nhs.uk/cor onavirus/wpcontent/uploads/sites/52/2020/03/C0044-SpecialtyGuide-Virtual-Working-and-Coronavirus-27-March-20.pdf

21. Mahase E (2020) Covid-19: hoarding and misuse of protective gear is jeopardising the response. WHO warns BMJ 368:m869
Publisher's Note Springer Nature remains neutral with regard to jurisdictional claims in published maps and institutional affiliations. 